\title{
Statistics of Real Eigenvalues in Ginibre's Ensemble of Random Real Matrices
}

\author{
Eugene Kanzieper ${ }^{b}$ and Gernot Akemann ${ }^{\sharp}$ \\ ${ }^{b}$ Department of Applied Mathematics, Holon Academic Institute of Technology, Holon 58102, Israel \\ ${ }^{\sharp}$ Department of Mathematical Sciences, Brunel University West London, Uxbridge UB8 3PH, United Kingdom
}

(Dated: 21 July 2005)

\begin{abstract}
The integrable structure of Ginibre's orthogonal ensemble of random matrices is looked at through the prism of the probability $p_{n, k}$ to find exactly $k$ real eigenvalues in the spectrum of an $n \times n$ real asymmetric Gaussian random matrix. The exact solution for the probability function $p_{n, k}$ is presented, and its remarkable connection to the theory of symmetric functions is revealed. An extension of the Dyson integration theorem is a key ingredient of the theory presented.
\end{abstract}

PACS numbers: 02.50.-r, 05.40.-a, 75.10.Nr

DOI: 10.1103/PhysRevLett.95.230201

Introduction.--In the mid-1960's, Ginibre introduced [1] statistical ensembles of (i) real, (ii) complex, and (iii) real quaternion random matrices whose eigenvalues may belong to any point of the complex plane $\mathbb{C}$. They were derived from the celebrated Gaussian orthogonal (GOE), Gaussian unitary (GUE), and Gaussian symplectic (GSE) random matrix ensembles [2] in a purely formal way by dropping the Hermiticity constraint and, therefore, can be thought of as their direct non-Hermitean descendants. Respectively coined as GinOE, GinUE, and GinSE, non-Hermitean random matrix models exhibited intriguingly rich mathematical structures whose complexity exceeded by far that of Hermitean random matrix theory (RMT).

From the physical point of view, non-Hermitean random matrices have proven to be as important [3] as their Hermitean counterparts [4]. Ginibre's random matrices appear in the description of dissipative quantum maps [5] (GinUE), in the studies of dynamics [6] and the synchronisation effect 7] in random networks (GinOE), in the statistical analysis of cross-hemisphere correlation matrix of the cortical electric activity [8] (GinOE), and in the characterisation of two-dimensional random space-filling cellular structures 9] (GinUE). They also arise in the context of "directed quantum chaos" 10, 11] (GinOE, GinUE, GinSE). Chiral deformations of non-Hermitean random matrices (GinOE, GinUE, GinSE) help elucidate universal aspects of the phenomenon of spontaneous chiral symmetry breaking in quantum chromodynamics [12]: the presence or absence of real eigenvalues singles out different breaking patterns. More recent findings [13] associate statistical models of non-Hermitean normal random matrices with integrable structures of conformal maps and interface dynamics at both classical [14] and quantum scales 15. For a comprehensive review of these and other physical applications, the reader is referred to Ref. 3].

Out of the three non-Hermitean matrix models 1, Ginibre's orthogonal ensemble defined by the probability density $P_{\text {GinOE }}[\mathcal{H}]=(2 \pi)^{-n^{2} / 2} \exp \left[-\operatorname{tr}\left(\mathcal{H}^{\mathrm{T}} / 2\right)\right]$ for an $n \times n$ real matrix $\mathcal{H}$ to occur is the least stud- ied and the most challenging. Perhaps, the great difficulties faced in statistical analysis of the GinOE can be attributed to the fact that its, generically complex, spectrum $\left(w_{1}, \cdots, w_{n}\right)$ may contain a finite fraction of real eigenvalues. This very peculiar feature of GinOE can conveniently be accommodated by dividing the entire space $\mathbb{T}(n)$ spanned by all real $n \times n$ matrices $\mathcal{H} \in \mathbb{T}(n)$ into $(n+1)$ mutually exclusive sectors $\mathbb{T}(n / k)$ associated with the matrices having exactly $k$ real eigenvalues, such that $\mathbb{T}(n)=\bigcup_{k=0}^{n} \mathbb{T}(n / k)$. The sectors $\mathbb{T}(n / k)$, characterised by the partial probability densities $P_{\mathcal{H} \in \mathbb{T}(n / k)}\left(w_{1}, \cdots, w_{n}\right)$, can be explored separately because they contribute additively to the joint probability density function (JPDF) of all $n$ eigenvalues of $\mathcal{H}$ from $\mathbb{T}(n)$ :

$$
P_{n}\left(w_{1}, \cdots, w_{n}\right)=\sum_{k=0}^{n} P_{\mathcal{H} \in \mathbb{T}(n / k)}\left(w_{1}, \cdots, w_{n}\right)
$$

Two spectral characteristics are of particular physical interest: (i) the partial $\left(r_{1}, r_{2}\right)$-point correlation functions obtained by integrating out all but $r_{1}$ real and $r_{2}$ complex eigenvalues in $P_{\mathcal{H} \in \mathbb{T}(n / k)}$, and (ii) the probability

$$
p_{n, k}=\prod_{i=1}^{k} \int_{\mathbb{R}} d \lambda_{i} \prod_{j=1}^{\ell} \int_{\mathbb{R}} d \operatorname{Re} z_{j} \int_{\mathbb{R}^{+}} d \operatorname{Im} z_{j} P_{\mathcal{H} \in \mathbb{T}(n / k)}
$$

to find exactly $k$ real eigenvalues in the spectrum of GinOE that additionally contains $\ell$ pairs of complex conjugated eigenvalues, so that $n=k+2 \ell$. The probability function $p_{n, k}$ is the central object of our study, which, if considered in a wider context, aims to highlight an exclusive rôle played by symmetric functions in the description of GinOE.

The most important and general result available for GinOE is due to the breakthrough work by Lehmann and Sommers [16] who proved, a quarter of a century after Ginibre's work, that the $k$-th partial JPDF $(0 \leq k \leq n)$ equals [17] 


$$
P_{\mathcal{H} \in \mathbb{T}(n / k)}=\frac{2^{\ell-n(n+1) / 4}}{i^{\ell} k ! \ell ! \prod_{j=1}^{n} \Gamma(j / 2)} \prod_{i>j=1}^{k}\left|\lambda_{i}-\lambda_{j}\right| \prod_{j=1}^{k} \prod_{i=1}^{\ell}\left(\lambda_{j}-z_{i}\right)\left(\lambda_{j}-\bar{z}_{i}\right) \prod_{j=1}^{k} e^{-\lambda_{j}^{2} / 2} \prod_{i>j=1}^{\ell}\left|z_{i}-z_{j}\right|^{2}\left|z_{i}-\bar{z}_{j}\right|^{2}
$$

$$
\times \prod_{j=1}^{\ell}\left(z_{j}-\bar{z}_{j}\right) \operatorname{erfc}\left(\frac{z_{j}-\bar{z}_{j}}{i \sqrt{2}}\right) e^{-\left(z_{j}^{2}+\bar{z}_{j}^{2}\right) / 2}
$$

In writing (3), we have used a representation due to Edelman [18] who rediscovered the result [16] a few years later. The above JPDF is supported for $\left(\lambda_{1}, \cdots, \lambda_{k}\right) \in$ $\mathbb{R}^{k},\left(\operatorname{Re} z_{1}, \cdots, \operatorname{Re} z_{\ell}\right) \in \mathbb{R}^{\ell}$, and $\left(\operatorname{Im} z_{1}, \cdots, \operatorname{Im} z_{\ell}\right) \in$ $\left(\mathbb{R}^{+}\right)^{\ell}$. A particular case $k=n$ of (3), corresponding to the matrices $\mathcal{H} \in \mathbb{T}(n / n)$ with all eigenvalues real, was first derived by Ginibre 1].

Equations (11) and (3) solve the problem of finding the JPDF of all $n$ eigenvalues in GinOE. Despite this tremendous progress, a calculation of the $\left(r_{1}, r_{2}\right)$-point correlation function based on (3) turns out to be the problem, particularly, because the well-developed machinery [2] of RMT, which was at use in studies of complex eigenvalue correlations in GinUE [1] and GinSE [19, 20], fails to work in this case. In particular, the famous Dyson integration theorem [21] (Theorem 5.1.4 in Ref. [2]) is no longer applicable to the study of spectral statistics in GinOE, as will be reasoned below. This failure clearly signals of novel mathematical structures lurking behind (3).

To unveil and explore these structures, we wish to concentrate on the probability function $p_{n, k}$. Previous attempts by Edelman and co-workers [18, 22] to attack the problem brought no explicit formula for $p_{n, k}$ for generic $k$. The analytic results currently available include: (i) the probability of having all $n$ eigenvalues real equals [18] $p_{n, n}=2^{-n(n-1) / 4}$ (this is the smallest probability out of all $p_{n, k}$ ); (ii) for all $0 \leq k \leq n$, the $p_{n, k}$ is of the form [18] $p_{n, k}=r_{n, k}+s_{n, k} \sqrt{2}$, where $r_{n, k}$ and $s_{n, k}$ are rational; (iii) the expected number $E_{n}=\sum_{k=0}^{n} k p_{n, k}$ of real eigenvalues is given by 22$]$

$$
E_{n}=\frac{1}{2}+\sqrt{2} \frac{{ }_{2} F_{1}(1,-1 / 2 ; n ; 1 / 2)}{B(n, 1 / 2)} .
$$

Main results. - The following four statements summarise the new results reported in this Letter.

Statement 1 . The probability $p_{n, k}$ of exactly $k$ real eigenvalues occurring equals

$$
p_{n, k}=p_{n, n-2 \ell}=p_{n, n} \mathcal{F}_{\ell}\left(p_{1}, \cdots, p_{\ell}\right) .
$$

The universal multivariate polynomials

$$
\mathcal{F}_{\ell}\left(p_{1}, \cdots, p_{\ell}\right)=(-1)^{\ell} \sum_{|\boldsymbol{\lambda}|=\ell} \prod_{j=1}^{g} \frac{1}{\sigma_{j} !}\left(-\frac{p_{\ell_{j}}}{\ell_{j}}\right)^{\sigma_{j}}
$$

are exclusively determined by the partitions $\boldsymbol{\lambda}$ of the size $|\boldsymbol{\lambda}|=\ell$, where $\ell$ is the number of pairs of complex-conjugated eigenvalues. The formula (6) uses a frequency representation [23] of those partitions $\boldsymbol{\lambda}=\left(\ell_{1}^{\sigma_{1}}, \cdots, \ell_{g}^{\sigma_{g}}\right)$. Experts in the theory of symmetric functions may readily recognise that our $\mathcal{F}_{\ell}$ 's are, up to a factorial, so-called zonal polynomials [24]

$$
\mathcal{F}_{\ell}\left(p_{1}, \cdots, p_{\ell}\right)=\frac{1}{\ell !} Z_{\left(1^{\ell}\right)}\left(p_{1}, \cdots, p_{\ell}\right) .
$$

They are tabulated in the manuscript 25] by Jack and can also be efficiently calculated 24 by recursion. On the contrary, the arguments $p_{j}$ 's of the multivariate polynomials in (5) are matrix model dependent:

$$
p_{j}=\operatorname{tr}_{(0,[n / 2]-1)} \hat{\varrho}^{j} \text {. }
$$

(The notation $[x]$ stands for the integer part of $x$ ). The non-universal matrix $\varrho$ is sensitive to the parity of $n$. For

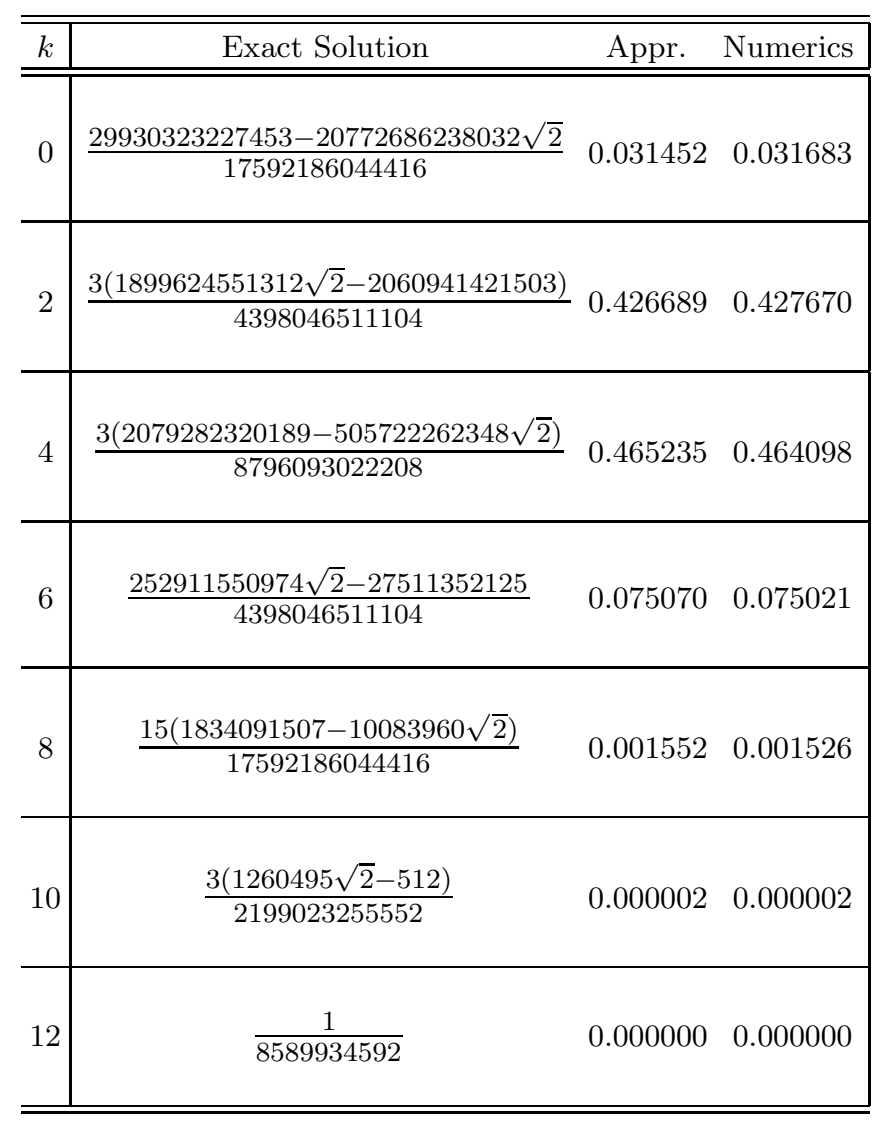

TABLE I: Exact solution for $p_{12, k}$ (second and third column) compared to numeric simulations (fourth column) performed by direct diagonalisation of $1,000,000$ of $12 \times 12$ matrices. 
$n=2 m$ even, its entries are

$$
\begin{aligned}
\hat{\varrho}_{\alpha, \beta}^{\text {even }} & =\int_{0}^{\infty} d y y^{2(\beta-\alpha)-1} e^{y^{2}} \operatorname{erfc}(y \sqrt{2})[(2 \alpha+1) \\
& \left.\times L_{2 \alpha+1}^{2(\beta-\alpha)-1}\left(-2 y^{2}\right)+2 y^{2} L_{2 \alpha-1}^{2(\beta-\alpha)+1}\left(-2 y^{2}\right)\right]
\end{aligned}
$$

while for $n=2 m+1$ odd,

$$
\hat{\varrho}_{\alpha, \beta}^{\text {odd }}=\hat{\varrho}_{\alpha, \beta}^{\text {even }}-(-4)^{m-\beta} \frac{m !}{(2 m) !} \frac{(2 \beta) !}{\beta !} \hat{\varrho}_{\alpha, m}^{\text {even }} .
$$

Here, $L_{j}^{\alpha}(x)$ are generalised Laguerre polynomials.

We wish to stress that zonal polynomials $Z_{\left(1^{\ell}\right)}$ are the integral part of the final solution (5), and not merely the means of its derivation. This highlights a strong connection between integrable structure of GinOE, integer partitions (6), and the theory of symmetric functions (7).

Statement 2. The entire generating function for the probabilities $p_{n, n-2 \ell}$ can be reconstructed from (5). The fairly compact result

$$
G_{n}(z)=\sum_{\ell=0}^{[n / 2]} z^{\ell} p_{n, n-2 \ell}=p_{n, n} \operatorname{det}\left(\mathbb{1}_{[n / 2]}+z \hat{\varrho}\right)
$$

with the $\hat{\varrho}$ of needed parity provides us with yet another way of computing the entire set of $p_{n, k}$ 's at once. For comparison of our analytic predictions with numeric simulations, see the Table I.

Statement 3. The previously unknown JPDF of $\ell$ pairs of complex conjugated eigenvalues of a matrix $\mathcal{H} \in \mathbb{T}(n / k)$ is given by the formula (15).

Statement 4. This statement is formulated in the form of the Pfaffian integration theorem (17) below. (It is highly likely that this theorem will have implications far beyond the scope of the present Letter).

Sketch of the derivation.-Because of the space limitations, only key points of the derivation will be presented below; the technical details will be reported elsewhere [26].

To determine the probability function $p_{n, k}$, defined by (21), we first integrate out all $\left(\lambda_{1}, \cdots, \lambda_{k}\right)$ in (3) which can be viewed as a hybrid of GOE [2] and GinSE [19, 20]. The most efficient way to do so is to spot that the first line in (3), if taken without any prefactors therein, is proportional to the average characteristic polynomial $\left\langle\prod_{j=1}^{\ell} \operatorname{det}\left(z_{j}-H\right) \operatorname{det}\left(\bar{z}_{j}-H\right)\right\rangle$ of a $k \times k$ random matrix $H$ drawn from the GOE with the weight function $\exp \left(-\operatorname{tr} H^{2} / 2\right)$. It admits the Pfaffian representation [27, 28]

$$
\frac{k !}{n !} \frac{c_{n} / c_{k}}{\Delta_{2 \ell}(\{z, \bar{z}\})} \operatorname{pf}\left[\begin{array}{ll}
K_{n}\left(z_{i}, z_{j}\right) & K_{n}\left(z_{i}, \bar{z}_{j}\right) \\
K_{n}\left(\bar{z}_{i}, z_{j}\right) & K_{n}\left(\bar{z}_{i}, \bar{z}_{j}\right)
\end{array}\right]_{2 \ell \times 2 \ell}
$$

with the constant $c_{k}=2^{k / 2} k ! \prod_{j=1}^{k} \Gamma(j / 2)$ given by a GOE Selberg's integral [2]. The Vandermonde determinant $\Delta_{2 \ell}(\{z, \bar{z}\})$ is calculated on the set of complex eigenvalues $\left(z_{1}, \cdots, z_{\ell} ; \bar{z}_{1}, \cdots, \bar{z}_{\ell}\right)$. The kernel function $K_{n}\left(z, z^{\prime}\right)$ in (12) is a so-called $D$-part 29] of the GOE matrix kernel [2] that can be expressed [30] in terms of arbitrary monic polynomials $q_{j}$ :

$$
K_{n}(x, y)=\frac{1}{2} \sum_{j, k=0}^{n-1} q_{j}(x) \hat{\mu}_{j k} q_{k}(y) .
$$

The real antisymmetric matrix $\hat{\mu}$ is determined by its inverse

$$
\left(\hat{\mu}^{-1}\right)_{j k}=\frac{1}{2} \int_{\mathbb{R}^{2}} d x d y e^{-\left(x^{2}+y^{2}\right) / 2} \operatorname{sgn}(y-x) q_{j}(x) q_{k}(y) .
$$

Substituting (12) into (2) completes the $\lambda$-integration therein, bringing a new result for the JPDF of $\ell$ pairs of complex conjugated eigenvalues of $\mathcal{H} \in \mathbb{T}(n / k)$ :

$$
P_{\mathcal{H} \in \mathbb{T}(n / k)}\left(z_{1}, \bar{z}_{1}, \cdots, z_{\ell}, \bar{z}_{\ell}\right)=\frac{p_{n, n}}{\ell !}\left(\frac{2}{i}\right)^{\ell} \prod_{j=1}^{\ell} \operatorname{erfc}\left(\frac{z_{j}-\bar{z}_{j}}{i \sqrt{2}}\right) e^{-\left(z_{j}^{2}+\bar{z}_{j}^{2}\right) / 2} \operatorname{pf}\left[\begin{array}{cc}
K_{n}\left(z_{i}, z_{j}\right) & K_{n}\left(z_{i}, \bar{z}_{j}\right) \\
K_{n}\left(\bar{z}_{i}, z_{j}\right) & K_{n}\left(\bar{z}_{i}, \bar{z}_{j}\right)
\end{array}\right]_{2 \ell \times 2 \ell} .
$$

The structure of (15) mirrors that of the JPDF of all complex eigenvalues in the GinSE (see, e.g., Chap. 15.2 in Ref. [2], and Ref. [20]), possibly triggering one to think that the remaining $z$-integrations in (2) could readily be accomplished by virtue of the Dyson integration theorem 2, 21]. Sadly, this is not the case because the $D$-part $K_{n}\left(z, z^{\prime}\right)$ of the GOE matrix kernel does not obey the projection property in the complex plane

$$
\int_{\operatorname{Im} w>0} d \alpha(w) K_{n}(z, \bar{w}) K_{n}\left(w, z^{\prime}\right) \neq \frac{1}{2} K_{n}\left(z, z^{\prime}\right)
$$

with respect to the measure $d \alpha(w)=\operatorname{erfc}[(w-\bar{w}) / i \sqrt{2}] \exp \left[-\left(w^{2}+\bar{w}^{2}\right) / 2\right] d^{2} w$ from (15) (compare to the Lemma 15.2.1 of Ref. 2]). Fortunately, the projection property is not actually necessary to carry out the integrations. We were able to prove the following Pfaffian integration theorem [26].

Theorem.- Let $d \alpha(z)$ be any benign measure on $z \in \mathbb{C}$, and the kernel function $K_{n}(x, y)$ be an antisymmetric 
function specified by (13). Then, the integration formula

$$
\left(\frac{2}{i}\right)^{\ell} \prod_{j=1}^{\ell} \int_{z_{j} \in \mathbb{C}} d \alpha\left(z_{j}\right) \operatorname{pf}\left[\begin{array}{ll}
K_{n}\left(z_{i}, z_{j}\right) & K_{n}\left(z_{i}, \bar{z}_{j}\right) \\
K_{n}\left(\bar{z}_{i}, z_{j}\right) & K_{n}\left(\bar{z}_{i}, \bar{z}_{j}\right)
\end{array}\right]_{2 \ell \times 2 \ell}=Z_{\left(1^{\ell}\right)}\left(\frac{1}{2} \operatorname{tr}_{(0, n-1)} \hat{\sigma}^{1}, \cdots, \frac{1}{2} \operatorname{tr}_{(0, n-1)} \hat{\sigma}^{\ell}\right)
$$

holds, provided the integrals in the l.h.s. exist.

The Pfaffian integration formula contains particular zonal polynomials 24] $Z_{\left(1^{\ell}\right)}$ whose arguments are traces of $\hat{\sigma}^{j}$ with

$$
\hat{\sigma}_{\alpha, \beta}=i \sum_{k=0}^{n-1} \hat{\mu}_{\alpha, k} \int d \alpha(z)\left[q_{k}(z) q_{\beta}(\bar{z})-q_{\beta}(z) q_{k}(\bar{z})\right] .
$$

Operationally, the proof [26] is based on a topological interpretation of the permutational expansion of Pfaffian in (17) combined with term-by-term integration.

Equipped with this theorem, we are ready to complete the $z$-integrations in (2). The calculation is most economic in the basis where monic polynomials $q_{j}$ 's coincide with GOE skew-orthogonal polynomials. For $n=2 m$ even, they are given by [2]

$$
\begin{aligned}
q_{2 j+1}(x) & =2^{-(2 j+1)} H_{2 j+1}(x)-2^{-(2 j-1)} j H_{2 j-1}(x), \\
q_{2 j}(x) & =2^{-2 j} H_{2 j}(x),
\end{aligned}
$$

so that

$$
\hat{\mu}_{j k}=\frac{1+(-1)^{k}}{2^{1-k} k ! \sqrt{\pi}} \delta_{j, k+1}-\frac{1+(-1)^{j}}{2^{1-j} j ! \sqrt{\pi}} \delta_{j, k-1} .
$$

Substituting (19) and (20) into (18), and taking into account (17), we reproduce the announced solution (5) (9) after a bit lengthy but straightforward calculations. The case $n=2 m+1$ odd can be treated similarly [26] leading to the same solution but with (9) replaced by (10).

It remains to establish the result (11) for the generating function $G_{n}(z)$. It stems from the summation formula

$$
\sum_{r=0}^{\infty} \frac{z^{r}}{r !} Z_{\left(1^{r}\right)}\left(p_{1}, \cdots, p_{r}\right)=\exp \left(\sum_{r \geq 1}(-1)^{r-1} \frac{p_{r} z^{r}}{r}\right)
$$

well known in the theory of symmetric functions 24]. Identifying $p_{r}=\operatorname{tr}_{(0,[n / 2]-1)} \hat{\varrho}^{r}$, the exponent in (21) can be evaluated explicitly to confirm (11).

Conclusion.-To summarise, the exact solution was presented for the probability $p_{n, k}$ to find precisely $k$ real eigenvalues in the spectrum of an $n \times n$ random matrix drawn from GinOE. Expressed in terms of zonal polynomials, the solution associates the integrable structure of GinOE with the theory of symmetric functions.

Certainly, more work is needed to accomplish the spectral theory of GinOE. (i) In particular, the large- $n$ behavior [26] of the probability function $p_{n, k}$ should be examined when the number $k$ of real eigenvalues does or does not scale with the matrix dimension $n$. The large$n$ formulae of this kind would facilitate a comparison of our exact theory with existing experimental [8] and numerical (see the second paper in Ref. [12]) data. (ii) The calculation of all $\left(r_{1}, r_{2}\right)$-point correlation functions for GinOE as defined below (11) is yet another important problem to tackle. We believe that progress in this direction can be achieved through a proper extension of the Pfaffian integration theorem (17).

We thank T. Seligman for kind hospitality at the Centro Internacional de Ciencias (CIC) in Cuernavaca, Mexico, where this work was initiated. Clarifying correspondence with A. Borodin (Caltech), V. B. Kuznetsov (Leeds) and G. Olshanski (IITP, Moscow) is greatly appreciated. A part of this work was done during the visits to Brunel University West London (E.K.) and University of Warwick (E.K. and G.A.) supported by a BRIEF grant from Brunel University. The research by E.K. was partially supported by the Israel Science Foundation through the grant No 286/04.

[1] J. Ginibre, J. Math. Phys. 6, 440 (1965).

[2] M. L. Mehta, Random Matrices, (Elsevier, New York, 2004), 3rd ed.

[3] Y. V. Fyodorov and H.-J. Sommers, J. Phys. A: Math. Gen. 36, 3303 (2003).

[4] T. Guhr, A. Müller-Groeling, H. A. Weidenmüller, Phys. Reports 299, 189 (1998).

[5] R. Grobe, F. Haake, and H.-J. Sommers, Phys. Rev. Lett. 61, 1899 (1988); R. Grobe and F. Haake, ibid. 62, 2893 (1989).

[6] H.-J. Sommers, A. Crisanti, H. Sompolinsky, and Y. Stein, Phys. Rev. Lett. 60, 1895 (1988); H. Sompolinsky, A. Crisanti, and H.-J. Sommers, ibid. 61, 259 (1988).

[7] M. Timme, F. Wolf, and T. Geisel, Phys. Rev. Lett. 89, 258701 (2002); M. Timme, F. Wolf, and T. Geisel, ibid. 92, 074101 (2004).

[8] J. Kwapień, S. Drożdż, and A. A. Ioannides, Phys. Rev. E 62, 5557 (2000).

[9] G. Le Caër and J. S. Ho, J. Phys. A: Math. Gen. 23, 3279 (1990); G. Le Caër and R. Delannay, J. Phys. I France 3, 1777 (1993).

[10] K. B. Efetov, Phys. Rev. Lett. 79, 491 (1997); Phys. Rev. B 56, 9630 (1997); A. V. Kolesnikov and K. B. Efetov, Waves Random Media 9, 71 (1999).

[11] Y. Fyodorov, B. Khoruzhenko, and H.-J. Sommers, Phys. Rev. Lett. 79, 557 (1997).

[12] M. Stephanov, Phys. Rev. Lett. 76, 4472 (1996); M. A. Halasz, J. C. Osborn, and J. J. M. Verbaarschot, Phys. 
Rev. D 56, 7059 (1997); H. Markum, R. Pullirsch, and T. Wettig, Phys. Rev. Lett. 83, 484 (1999); G. Akemann and T. Wettig, ibid. 92, 102002 (2004).

[13] A. Zabrodin, in Proceedings of the International Conference on Theoretical Physics (TH-2002), edited by D. Iagolnitzer, V. Rivasseau, and J. Zinn-Justin (Birkhäuser Verlag, Basel, 2003).

[14] M. Mineev-Weinstein, P. B. Wiegmann, and A. Zabrodin, Phys. Rev. Lett. 84, 5106 (2000).

[15] O. Agam, E. Bettelheim, P. Wiegmann, and A. Zabrodin, Phys. Rev. Lett. 88, 236801 (2002).

[16] N. Lehmann and H.-J. Sommers, Phys. Rev. Lett. 67, 941 (1991).

[17] Throughout the paper, we use a parameterisation $\left(w_{1}, \cdots, w_{n}\right)=\left(\lambda_{1}, \cdots, \lambda_{k} ; z_{1}, \bar{z}_{1}, \cdots, z_{\ell}, \bar{z}_{\ell}\right)$.

[18] A. Edelman, J. Multivar. Analysis 60, 203 (1997).

[19] M. L. Mehta and P. K. Srivastava, J. Math. Phys. 7, 341 (1966).

[20] E. Kanzieper, J. Phys. A: Math. Gen. 35, 6631 (2002).

[21] F. J. Dyson, Commun. Math. Phys. 19, 235 (1970).

[22] A. Edelman, E. Kostlan, and M. Shub, J. Am. Math. Soc. 7, 247 (1994).

[23] A partition $\boldsymbol{\lambda}$ of the size $|\boldsymbol{\lambda}|=\ell$ written in the frequency representation $\boldsymbol{\lambda}=\left(\ell_{1}^{\sigma_{1}}, \cdots, \ell_{g}^{\sigma_{g}}\right)$ implies that the part $\ell_{j}$ appears $\sigma_{j}$ times so that $\ell=\sum_{j=1}^{g} \ell_{j} \sigma_{j}$, where $g$ is the number of inequivalent parts of partition.

[24] I. G. Macdonald, Symmetric Functions and Hall Polynomials (Oxford University Press, New York, 1998), 2nd ed.

[25] H. Jack, "A Class of Polynomials in Search of a Definition, or the Symmetric Group Parameterized", in: Proceedings of the Workshop on Jack, Hall-Littlewood and Macdonald Polynomials, edited by V. B. Kuznetsov and S. Sahi (American Mathematical Society, Providence, RI, to be published).

[26] E. Kanzieper and G. Akemann, unpublished (2005).

[27] T. Nagao and S. M. Nishigaki, Phys. Rev. D 63, 045011 (2001).

[28] A. Borodin and E. Strahov, Commun. Pure and Appl. Math. 58, 0001 (2005).

[29] C. A. Tracy and H. Widom, J. Stat. Phys. 92, 809 (1998).

[30] Strictly speaking, the formulae (13) and (14) as they stand are only valid for $n=2 m$ even. Since for $n=2 m+1$ odd a similar representation is available [2, 27] for the kernel $K_{n}(x, y)$, our derivation holds for $n$ of any parity. 